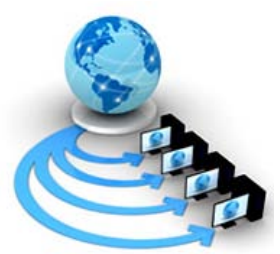

Volume 12, No. 1, January-February 2021

International Journal of Advanced Research in Computer Science

REVIEW ARTICLE

Available Online at www.ijarcs.info

\title{
MOBILITY MANAGEMENT IN RADIO BASED OPTIMIZED NETWORKS
}

\author{
Putri Kevin \\ Department of Computer Science, \\ Brahijaya University, \\ Indonesia
}

\author{
Dian viely \\ Department of Computer Science, \\ Brahijaya University, \\ Indonesia
}

\begin{abstract}
In recent years, the development in WSN is exponentially growing. Recently, there has been a lot of emphasis on mobile sensor networks, capable of monitoring their movements. Mobility attenuates problems in coverage and connection in the network. This paper profoundly surveys on localization and taxonomy of mobile sensors.
\end{abstract}

Keywords: Mobility, Wireless sensor network, path planning

\section{INTRODUCTION}

WSN never visualize as static; mobility was having many issues like connectivity issues, coverage, and energy utilization that needed to control. Through mobility, the sensor can track the moving targets, i.e., Vehicles. Localization is a challenging issue in MWSN. The NAVSTAR Global Positioning System (GPS) is currently the most widely used method for localization. GPS with 24 satellites revolving around the earth, four of them are using for location. The satellite transmits a signal consisting of a data message and a mobile antenna that signals to compute location [1-4].

GPS (Commercial Use) accuracy is about within $10 \mathrm{~m}$. Gps is limited in some applications like indoor, underwater water, and MWSN in urban deployment. GPS Less localization is the main aim of this research [5-7].

\section{MOBILE BASED WSN}

In this section, we explain the taxonomy of MWSN and compare the MWSN and WSN. Mobility advantages.

\section{A. Mobile based WSN Architechture}

Mobile sensor networks can be classified as hierarchical architecture as flat, 2-Tier, or 3-Tier. The flat network consists of collecting devices that communicate over the same network in an ad hoc manner. Devices may be stationary and mobile. The two-tier network comprises both stationary and mobile nodes. The mobile nodes help in data transfer through an overlay-network [8-11]. The mobile devices have excellent processing, communication range, and have greater bandwidth. Overlay network density is such that all nodes are connected, or the network may disconnect. In the third tier, the network consists of sets of stationary nodes and groups of mobile nodes. A collection of static nodes sends packets of data to mobile nodes that forwarded this to the access point. This network type is a heterogeneous network designed to cover a large region and compact with no application immediately. i.e., Parking Area Monstering sensor. The mobile sensor classifieds based on their usage innetwork at the node level [12].

Mobile Embedded Sensor: Mobile embedded nodes do not regulate their movement; instead, their motion is guided by an external force, such as being connected to an animal or attached to a shipping container [13-14].

Mobile Actuated Sensor: Sensor nodes have a moving ability that enables them to move around the sensor sensing region. This ability makes deployment specification become more precise and maximize the coverage.

Data Mule: Sensors require mobile, may it require a mobile device to gather information and send information to a base station. This type of operator is known as data mules.

Access Point: when a node drops off the network, mobile nodes may be placed to retain network access. In this situation, they serve as an access point network [15-18].

\section{B. Advantage of adding mobility}

A node may be placed in a grid manner around the object of interest and many arrangements. Optimal implementation remains unknown in many cases till nodes of the sensor start gathering and processing data. The rearrangement is not possible in remote area deployment. Redeployment is only feasible when nodes are mobile. Mobile in WSNs increases the coverage and use of the network deployment sensor [19].

When stationary nodes die, the mobile nodes come in to link the communication route. In WSN, static data lost when the static node fails [20]. When network sinks are stationary, nodes closer to the base station will die sooner because they must send more data than farther nodes. Mobility also allows improved channel capacity and protects data confidentiality by creating multiple contact routes and reducing the number of hop messages to traverse before reaching their destination [21-25].

\section{WSNs and MWSNs}

Localization: Mobile nodes always getting their position; this needs more time and energy while the static node position is computed only at an initial stage.

Dynamic Network Topology: In dynamic network topologies, table data is quickly out of date and route. The finding must be made repeatedly at a significant expense in power, time, and bandwidth.

Power Consumption: MWSN needs extra mobility power and is often fitted with a more excellent battery source or self-charging capability that allows them to plug into the energy source grid to charge their batteries[26].

Network Sink: In centralize WSN, data is sent to a base station, where it is analyzed. Data routing and aggregation 
can lead to high overhead costs. Few MWSNs uses mobile base stations that cross through the sensing area to gather data or position themselves in such a way that the number of transmitting hops is reduced sensor nodes.

\section{Positioning In MWSNs}

In this section, we use 3 phases of MWSN (1) coordination, (2) measurement, and (3) position estimation. They coordinate a community of nodes to begin the localization. One or more nodes emit a signal, and some of the signal properties (e.g., time of arrival, phase, signal power, etc.) are detected by one or more than one receiver. The node's location is then determined by transformation measurement of signals in location estimates through a localization algorithm [27].

To find the position, it is often necessary to enlist the help of cooperating sensor nodes that have been deployed into the environment at known positions a priori. These devices are referred to as anchor, infrastructure, or seed nodes. For example, in GPS, the infrastructure nodes are the satellites that orbit the planet [28]. The position estimate may be relative to a set of stationary anchor nodes at known positions in a local coordinate system, or absolute coordinates may be obtained if the anchor nodes' positions are known to some global coordinate system (i.e., using GPS).

referred to as anchor, infrastructure, or seed nodes. For example, in GPS, the infrastructure nodes are the satellites that orbit the planet. The position estimate may be relative to a set of stationary anchor nodes at known positions in a local coordinate system, or absolute coordinates may be obtained if the anchor nodes' positions are known to some global coordinate system (i.e., using GPS) [29].

\section{A. Coordination Phase}

In localization before signal transmission, the nodes coordinate with each other. Such coordination, which notifies that the localization phase is about to start, and clock synchronization, helps the obtained signal data be processed within a popular timeline. These coordination methods are of microsecond precision and involve the delivery of only one Packet (Message) [2]. The message encoded is the timestamp of the message sender introduced into the message directly before the delivery, minimizing the aggregate of nondeterministic delay implied in the synchronization. Within the broadcast spectrum, all nodes will receive the message simultaneously and, assuming a marginal transmission time of the radio signal through the air, and it will be able to translate the timestamp of the transmitter to the local timescale [30].

\section{B. Measurement phase}

WSNs are configured to have low-cost wide-area observation ability, and it is usually not desirable to add additional hardware to the sensor board since this raises cost and power usage. For Example, In humid conditions, radio signals work weaker than acoustic signals due to the humidity in the air reflects and absorbs high-frequency radio waves but do not affect vibrational sound waves. For example, the military application uses radio frequency than the acoustic [31].

The acoustic includes an ultrasound and audible sound wave propagation. Active bats and crickets are mostly used in localization approaches. An ultrasound technique, including an ultrasound survey positioning systems and problems, can be described in. Many localization techniques have been evolved in an acoustic band, i.e., sniper tracking and detection system. IR signals have great noise and attenuation. IR works only when the receiver and transmitter close to each other. This is appropriate for most indoor position systems, but not for the outdoor location. It is difficult not just because of proximity problems but also because IR is not readable during sunlight.

Since all wireless sensor nodes have radio hardware, RF propagation has become a common signal modality for localization. Signal power, phase, frequency, and strength are evaluated to measure the spectrum of position estimation data. One advantage of using RF is that it has been shown to achieve localization precision in centimeters, often in a sparse network.

Lighthouse and Spotlight localization strategies use illumination beacon to decide the location of the node. While both approaches assert high precision and accuracy, they require a LOS, a strong light source that works well in the lighted areas, and upgradable light source hardware.

Traditional sensor node radios communicate between $400 \mathrm{MHz}$ and $2.6 \mathrm{GHz}$, and sampling frequency cannot be achieved with resource-restricted hardware. The beat signal's frequency and phase will then be determined by observing the obtained signal intensity indicator on-chip to produce a low-frequency beat signal.

\section{Measurement Techniques}

Several methods are based on signal measurement, exits for getting bearing, proximity, and range. An angle-ofarrival (AOA) approach requires the calculation of the angular separation between an individual or more than two and a fixed axis. The place can be calculated by setting the AOA at a certain number of sensor nodes. The techniques of angulation determined position. TOA localization is based on the time taken by the signal to reach the sensor node. TOA consists of coordination between transmitter and receiver. The only downside to this strategy is that it is impossible to reliably record the arrival of the radio waves when they fly close to the speed of light. It fits nicely for an acoustic source [32]. TDOA is an improved TOA version that removes the need to know the information when a signal was sent. Several time-synchronized nodes receive a signal and look at the difference in arrival time at a particular time moment. As the signal moves at a uniform speed, the direction of sources easily calculated if enough nodes are engaging.

As the inverse-square theorem regulates the free-space signal intensity model, effective localization is possible. Besides, this usually does not require any hardware adjustments, and most chips allow program access to received signal amplitude. An RSS is a profile in which a chart of RSS values is built during the initial training process. Sensors then approximate their location by comparing the measured RSS values to the training results. Another approach is doppler shift, in which node position is determined by relation motion between sender and receiver, the signal frequency-shifts.

\section{Localization Calculation phase}

Signal data from the measurement phase may be used to determine the estimated position of a target node.

Lateration. For 2D localization, there are three measurement ranges required. Anchor acts as a center of the 
circle, and range acts as a radius. Three circles intersect at a point; that point is the target node location.

Angulation: For triangulation, two anchor node is used to define as third point in triangular of two known angles and one side. Two or more a bearing can be used, and the intersection of bearings computes the target position. All bearing is not intersecting at a common point, it may have a noise margin, but the target node lies in that region where bearing intersect.

Cellular Proximity. The range-free approach under which a node is localized in the area in which it is observed. This approach usually gives a more reliable estimation of the position, which relies on nodes' density [33].

Dead Reckoning: Robots shall receive their current speed from wheel encoders or other sources and shall use this knowledge following the period passed after the previous change to derive the current direction and heading. The downside of this approach is that the position calculation results in an error, most commonly due to the encoder's faulty reading due to irregular surface, wheel alignment, etc.

Estimation Methods: The state estimation method is used when data is noisy and the system undefine. MLE is a maximum like hood estimation approach that computes state value based on measured data, and no previous knowledge on the state is used, and no initial knowledge of state is used. SBE is a sequential Bayesian estimation that uses its initial knowledge to compute the state value on a datameasured basis. MLE chooses system parameters that make the observed data "Likely" than other values for the parameters. The likelihood data is determined using a calculation mechanism that compares the observed data to the system state. The Bayes rule calculates the system state in SBE. This explains that the following is proportional to the data likelihood and the expected posterior. Like MLE, the data probability is determined using a calculation model. The solution to SBE is not analytically calculated many optimal solution exist like Kalman Filter, Extended Kalman Filter (EKF), and Particle Filters. (PF) As the number of particles grows to be very large, the particle filter is approaching an optimal solution [34].

\section{E. Mobility effect on localization}

Usually, the positioning of mobile sensors is done to detect them. Latency is one major issue, and If the time taken to execute the localization is too long, the sensor would have dramatically shifted its location before the calculation took place. For example, in a robot navigation system, the robot moves with $1 \mathrm{~m} / \mathrm{s}$, and the algorithm takes $5 \mathrm{~m} / \mathrm{s}$ for computing measurements, and the robot moves $5 \mathrm{~m}$ away from the expected position. Mobility directly effects on localization signal. Error is introducing in calculation due to the doppler shift in the signal. Doppler shift is the relation between the relative motion of the sender and receiver. The resulting frequency shift is related to the direction and relative speed of the two nodes. Doppler effect uses to find out the velocity and position. If the localization strategy includes a (LOS), there is a risk that the mobile sensor may switch from a location with a strong LOS to a position with a weak LOS. There is LOS in the network of the mobile node whenever it moves.

\section{F. Centralized vs distributed algorithms}

The centralized algorithm runs on the base station; all nodes in the network onward the data to the base station.
The benefit of a centralized solution is that an algorithm can be built that is more precise, more accurate, and can handle larger volumes of data [32]. A centralized algorithm's drawbacks are that base station processing undergoes low scalability, difficult data routing, and maximum power utilization. Mobility needs constant and accelerated localization. While the centralized localization approach is for mobile sensors, but in navigation, it's not fast. The latency of distribution is nearly one second while centralize about 5 sec.

\section{G. The Impact of environment on Localization}

The environment plays a key role in the reliability of the localization approach. Consequently, there is no single localization process that will be effective in any of the cases. Various environmental variables are described below and the effects they have on the localization approaches referred to above. Crystal oscillators of the transceiver are directly affected by ambient temperature, pressure, and humidity, impacting localization accuracy. Radio waves are affected by humidity in the air. The localization RF approach can be hindered under these circumstances.

The most common problem is that GPS is not working underwater or indoor and in bad weather conditions. Indoor localization fails due to multipath lost factor.

\section{MWSNS APPLICATION WITH LOCALIZATION}

\section{A. Commercial surveys in industries}

The skilligent companies are designing and developing a software protocol for robots to perform nursing to care for a patient at home, security, and repairing different things in offices. skillget uses pattern matching visual localization approach. Objects are recorded in the system as landmarks. The robot knows its location by comparing or matching videos/images of recorded objects [35-36].

\section{B. Housekeeping}

Roomba is a vacuum cleaner robot that automatically scans the room. When it moves around, sing feedback from jumps \& optical sensors to the room, and then start cleaning. After cleaning the room, Roomba automatically returns to the charging pod.

\section{Environmental factor}

MWSN have great importance in tracking environment like in remote area to collect data.

\section{Wildlife tracking}

ZebraNet is an MWSN in which zebras were equipped with wireless devices to monitor their movement. Due to no cellular network coverage in the Remote area, data were redirected to mobile base stations via peer-to-peer networks. Zebras have not been limited to those regions, other than small devices mounted to their skins, and they were left undisturbed.

\section{E. Pollution Monitoring}

Sensor nodes are mounted on cars as cars derive along the highway's sensor records air quality, and concentration of different air containments present in the air and different location and time. When the sensors are placed in the access points' vicinity, the data is uploaded to the registry and released on the server [37]. 


\section{F. Civil}

This covers some non-military applications that sustain society on going smoothly and safely.

\section{G. Pothole Detection}

A device to identify the potholes in the city street is developed. The sensor is mounted on a local cab service in the city, which has an accelerometer and connects with WLAN or cellular data [38].

\section{H. Wireless E-911}

In America, E-911 is an emergency services number that connects the caller to emergency services members to link phone caller location. A new version of E11 commissioned by FCC needs a wireless cellular device to arrange the caller's location when service is requested automatically. Its deployment is non-trivial, and various carriers tend to use different approaches, including built-in GPS chips, multilateration, and angulation, depending on the cell tower's known location [39].

\section{Military/Aerospace}

Localization got attention in military applications like surveillance and navigation of the friendly and hostile individual. Extensive research in that field is going on.

\section{J. Shooter/weapon detection}

Soldier wearable sensor, which is mounted on soldier helmet to detect the enemy sniper's location and type of weapon they are using based on TDOA, acoustic properties of signals are used to survey enemies' position and analyze the weapon type [40-41].

\section{K. Deployment}

Unmanned aerial vehicles deploy sensors, which help the military in battlefield surveillance and command \& control operation.

\section{Conculusion}

Localization in MWSNs poses new problems arising from the deployment of resource-constrained wireless sensors on a mobile network. The localization methods have greater accuracy and precision on a mobile system with less limitation of resources are not appropriate. The static localization approach is unacceptable for most MWSN applications. Minimize the latency of localization is one of the critical specifications in MWSN. There is currently a tradeoff between the quick operation of an algorithm and its precision. Additional analysis is required to reduce run-time lag while retaining positioning accuracy. Most of the localization algorithm approaches are centralized. This is the worst design issue for the mobile location because of the extra energy and latency. Localization gets a lot of attention in an urban and indoor environment, where obstacle like furniture, people, and walls causes the multipath and LOS loss. Many existing approaches use RSS profiling variants in combination with optimization techniques. New approaches are needed to extend mobile sensing to locations where training data are not collected safely, such as urban conflict zones or burning houses. Mobile actuated sensors are being built with motorized form factors type. Like built-in sensor nodes (Embedded Sensor), these systems often have a resource hindrance that restricts their ability to traverse the sensing area in the same way as a robot with a wide range of sensors and a robust computing capability may be added. We hope for many innovations and advancements in the future in mobile sensing navigation.

\section{REFERENCES}

[1] Shule, Wang, et al. "Uwb-based localization for multiuav svstems and collaborative heterogeneous multirobot systems: a survey." arXiv preprint arXiv:2004.08174 (2020).

[2] Ahmad, T., Li, X. J., \& Seet, B. C. (2017). Parametric loop division for 3D localization in wireless sensor networks. Sensors, 17(7), 1697.

[3] L. Qingqing et al. Visual Odometry Offloading in Internet of Vehicles with Compression at the Edge of the Network. In 12th ICMU, 2019.

[4] W. Kong et al. Autonomous landing of an uav with a ground-based actuated infrared stereo vision system. In IEEE/RSJ IROS. IEEE, 2013.

[5] R. Faragher et al. An analysis of the accuracy of bluetooth low energy for indoor positioning applications. In 27th ION GNSS+), 2014.

[6] W Stempfhuber and M Buchholz. A precise, low-cost rtk gnss system for uav applications. Unmanned Aerial Vehicle in Geomatics, 2011.

[7] J. Q. Cui et al. Drones for cooperative search and rescue in post-disaster situation. In 7th CIS-RAM. IEEE, 2015.

[8] V. Lesser and D. Corkill, "Functionally accurate, cooperative dis-tributed systems,” IEEE Trans. Syst., Man, Cybern., vol. 11, pp. 81-96, Jan./Feb. 1981.

[9] R. B. Wesson, F. A. Hayes-Roth, J. W. Burge, C. Stasz, and C. A. Sunshine, "Network structures for distributed situation assessment,” IEEE Trans. Syst., Man, Cybern., vol. SMC-11, pp. 5-23, Jan./Feb. 1981.

[10] R. Rashid and G. Robertson, “Accent: A communication oriented network operating system kernel," in Proc. 8th Symp. Operating System Principles, 1981, pp. 64-75.

[11] R. Rashid, D. Julin, D. Orr, R. Sanzi, R. Baron, A. Forin, D. Golub, and M. Jones, "Mach: A system software kernel," in 34th Computer Society Int. Conf. (COMPCON), San Francisco, CA, 1989.

[12] C. Myers, A. Oppenheim, R. Davis, and W. Dove, "Knowledge-based speec analysis and enhancement," presented at the Int. Conf. Acoustics, Speech and Signal Processing, San Diego, CA, 1984.

[13] S. S. Kulkarni, "TDMA Services for Sensor Networks,"Proc. 24th Int'l. Conf. Distrib. Comp. Sys. Wksps., Mar.2004, pp. 604-09.

[14] W. Ye, J. Heidemann, and D. Estrin, "Medium Access Control with Coordinated Adaptive Sleeping for Wireless Sensor Networks,” IEEE/ACM Trans. Net., vol. 12, no. 3, June 2004, pp. 493-506.

[15] A. El-Hoiydi, "Spatial TDMA and CSMA with Preamble Sampling for Low Power Ad Hoc Wireless Sensor Networks,” Proc. ISCC 2002, July 2002, pp. 685-92.

[16] V. Rajendran, K. Obraczka, and J. J. Garcia-LunaAceves, "Energy-Efficient, Collision-Free Medium Access Control for Wireless Sensor Networks,” Proc. 
ACM SenSys '03, Los Angeles, CA, Nov. 2003, pp. 181-92.

[17] L. Bao and J. J. Garcia-Luna-Aceves, “A New Approach to Channel Access Scheduling for Ad Hoc Networks," 7th Ann. Int'l. Conf. Mobile Comp. and Net., 2001, pp. 210- 21.

[18] Ahmad, Tanveer, Xue Jun Li, and Boon-Chong Seet. "A self-calibrated centroid localization algorithm for indoor ZigBee WSNs." In 2016 8th IEEE International Conference on Communication Software and Networks (ICCSN), pp. 455-461. IEEE, 2016.

[19] Dawadi, B. R., Rawat, D. B., Joshi, S. R., \& Keitsch, M. M. (2020). Towards energy efficiency and green network infrastructure deployment in Nepal using software defined IPv6 network paradigm. The Electronic Journal of Information Systems in Developing Countries, 86(1), e12114.

[20] Ahmad, Tanveer, Xue Jun Li, and Boon-Chong Seet. "3D localization based on parametric loop division and subdivision surfaces for wireless sensor networks." In 2016 25th Wireless and Optical Communication Conference (WOCC), pp. 1-6. IEEE, 2016.

[21] K. Jamieson, H. Balakrishnan, and Y. C. Tay, "Sift: A MAC Protocol for Event-Driven Wireless Sensor Networks,” MIT Lab. Comp. Sci., Tech. rep. 894, May 2003, available http://www.lcs.mit.edu/publications/pubs/pdf/MITLCSTR-894.pdf

[22] Y. C. Tay, K. Jamieson, and H. Balakrishnan, "CollisionMinimizing CSMA and Its Applications to Wireless Sensor Networks,” IEEE JSAC, vol. 22, no. 6, Aug. 2004, pp. 1048-57.

[23] Ahmad, T., Li, X. J., Wenchao, J., \& Ghaffar, A. (2020, September). Frugal Sensing: A Novel approach of Mobile Sensor Network Localization based on FuzzvLogic. In Proceedings of the ACM MobiArch 2020 The 15th Workshop on Mobility in the Evolving Internet Architecture (pp. 8-15).

[24] Ahmad, Tanveer, Xue Jun Li, and Boon-Chong Seet. "3D localization using social network analysis for wireless sensor networks." In 2018 IEEE 3rd international conference on communication and information systems (ICCIS), pp. 88-92. IEEE, 2018.

[25] Ahmad, T., Li, X. J., \& Seet, B. C. (2017). Parametric loop division for 3D localization in wireless sensor networks. Sensors, 17(7), 1697.

[26] Chen, Hongvang, Pei Huang. Marcelo Martins, Hing Cheung So, and Kaoru Sezaki. "Novel centroid localization algorithm for three-dimensional wireless sensor networks." In 2008 4th International Conference on Wireless Communications. Networking and Mobile Computing, pp. 1-4. IEEE, 2008.

[27] Chen, Kai, Zhong-hua Wang. Mei Lin, and Min Yu. "An improved DV-Hop localization algorithm for wireless sensor networks." (2010): 255-259.

[28] Ahmad, T. (2019). 3D Localization Techniques for Wireless Sensor Networks (Doctoral dissertation, Auckland University of Technology).

[29] zeng Wang, Ji, and Hongxu Jin. "Improvement on APIT localization algorithms for wireless sensor networks."
In 2009 International Conference on Networks Security, Wireless Communications and Trusted Computing, vol. 1, pp. 719-723. IEEE, 2009.

[30] Ahmad, Tanveer, Xue Jun Li, and Boon-Chong Seet. "Noise Reduction Scheme for Parametric Loop Division 3D Wireless Localization Algorithm Based on Extended Kalman Filtering." Journal of Sensor and Actuator Networks 8, no. 2 (2019): 24.

[31] Ahmad, Tanveer, Xue Jun Li, and Boon-Chong Seet. "Fuzzy-Logic Based Localization for Mobile Sensor Networks." In 2019 2nd International Conference on Communication, Computing and Digital systems (CCODE), pp. 43-47. IEEE, 2019.

[32] G. Lu, B. Krishnamachari, and C. S. Raghavendra, “An Adaptive Energy-Efficient and Low-Latency MAC for Data Gathering in Wireless Sensor Networks," Proc. 18th Int'l. Parallel and Distrib. Processing Symp., Apr. 2004, p. 224.

[33] Ahmad, Tanveer, Xue Jun Li, Boon-Chong Seet, and Juan-Carlos Cano. "Social Network Analysis Based Localization Technique with Clustered Closeness Centrality for 3D Wireless Sensor Networks." Electronics 9, no. 5 (2020): 738.

[34] hakila, R., and B. Paramasivan. "An improved range based localization using Whale Optimization Algorithm in underwater wireless sensor network." Journal of Ambient Intelligence and Humanized Computing (2020): 1-11.

[35] K. Lu, X. Xiang, D. Zhang, R. Mao, and Y. Feng, "Localization algorithm based on maximum a posteriori in wireless sensor networks," International Journal of Distributed Sensor Networks, vol. 2012, Article ID 260302, 7 pages, 2012.

[36] J. Lee, W. Chung, and E. Kim, "Robust DV-Hop algorithm for localization in wireless sensor network," in Proceedings of the International Conference on Control, Automation and Systems, pp. 2506-2509, Gyeonggi-do, South Korea, October 2010.

[37] Ware, R.; Lad, F. Approximating the Distribution for Sum of Product of Normal Variables; Research report; the Mathematics and Statistics department at Canterbury University: Christchurch, New Zealand, 2003.

[38] Wen, C.-Y.; Hsiao, Y.-C. Decentralized anchor-free localization for wireless ad-hoc sensor networks. In Proceedings of IEEE International Conference on Systems, Man, and Cybernetics, Singapore, October 2008; pp. 2777-2785.

[39] Roberts, G.; Gelman, A.; Gilks, W. Weak Convergence and Optimal Scaling of Random Walk Metropolis Algorithms. Technical Report; University of Cambridge: Cambridge, UK, 1994.

[40] Chan, Fu and Chih-yu "Adaptive AoA/ToA localziation using fuzzy particle for mobile WSNs" in 2011 IEEE $73^{\text {rd }}$ VTC Spring 2011.

[41] F. Zhao, J. Shin, and J. Reich, "Information-driven dynamic sensor collaboration for tracking applications," IEEE Signal Processing Mag., vol. 19, pp. 61-72, Mar. 2020. 